\title{
A Program for Optimizing the Use of Antibiotics in the Hospital Setting: Experience in a Spanish Regional Hospital
}

\author{
Jon Ugalde-Espiñeira ${ }^{1^{*}}$, Jaione Bilbao-Aguirregomezcorta ${ }^{2}$ and Ainhoa Zuriñe Sanjuan-López ${ }^{1}$ \\ ${ }^{1}$ Service of Internal Medicine, Hospital San Eloy, Integrated Health Care Organization, Barakaldo-Sestao, Barakaldo, Spain \\ ${ }^{2}$ Service of Hospital Pharmacy, Hospital San Eloy, Integrated Health Care Organization Barakaldo-Sestao, Barakaldo, Spain
}

"Corresponding author: Jon Ugalde Espiñeira, MD, Servicio de Medicina Interna, Hospital San Eloy, Organización Sanitaria Integrada Barakaldo-Sestao, Avda Antonio Miranda 5, E-48902, Barakaldo, Spain, Tel: +34 94 4006700; Fax: +34 94 4006727; E-mail: j.ugalde.espineira@osakidetza.eus

Received date: October 03, 2016; Accepted date: October 31, 2016; Published date: November 7, 2016

Copyright: (c) 2016 Ugalde-Espiñeira J, et al. This is an open-access article distributed under the terms of the Creative Commons Attribution License, which permits unrestricted use, distribution, and reproduction in any medium, provided the original author and source are credited.

\begin{abstract}
We here describe the implementation of a program for optimizing the use of antibiotics (PROA) in a 128-bed regional hospital in Barakaldo, Spain, and the results obtained at 6 months. The study was a quasi-experimental prospective intervention study using a historical control group. A non-restrictive intervention model to help prescription, with a direct and bidirectional intervention was developed. The program consisted of an optimization audit of the use of antibiotics without pre-established personalized guidelines. Variables assessed included antibiotic consumption and costs, cost per process, mean hospital stay, and percentage of hospital readmissions. Data obtained during the 6-month study period (November 2013-April 2014) were compared with data collected between November 2012 and April 2013. A total of 307 audits were performed. In $65.8 \%$ of cases, treatment was discontinued between the $7^{\text {th }}$ and the $10^{\text {th }}$ day. The main reasons of treatment discontinuation were completeness of treatment $(43.6 \%)$ and lack of indication $(14.7 \%)$. The reduction of pharmaceutical expenditure was $8.59 \%$ $(P=0.049)$ and $5.61 \%$ of the consumption in defined daily dose (DDD)/100 stays $(P=0.180)$. The costs by processes in general surgery showed a $3.14 \%$ decrease $(P=0.001)$. These results confirm the efficiency of implementing programs for optimizing the use of antibiotics in medium-sized hospitals with limited resources.
\end{abstract}

Keywords: $\quad$ Antibiotics; Drug utilization; Medical audit; Quality indicators; Health care

\section{Background}

Rational use of antibiotics, maximizing the therapeutic efficacy of treatment and minimizing the risks associated with emerging infections and the selection of resistant pathogens, is widely recognized as an integral part of good clinical practice [1]. The indiscriminate and excess use of antimicrobial drugs appears the most significant factor in the emergence of resistant microorganisms in recent years. However, policies to promote better use of antibiotics in the hospital setting have been extensively recommended but resistant bacteria have persisted despite the theoretical benefits of different strategies, including restriction of antimicrobials, cycling and mixing approaches, and the use of combination therapies [2]. However, due to the large degree of inappropriate antimicrobial use, the impact that all usage has on resistance, superinfections, and safety and the negative impact of infections caused by resistant microorganisms and Clostridium difficile on patient outcomes, national initiatives have been implemented to optimize antibiotic use.

In Spain, the Hospital Infection Working Group-Spanish Society of Infectious Diseases and Clinical Microbiology (GEIH-SEIMC), Spanish Society of Hospital Pharmacy (SEFH) and the Spanish Society of Preventive Medicine, Public Health and Hygiene (SEMPSPH), promoted the development of a consensus document for the implementation of programs for optimizing the use of antibiotics in hospitals (PROA) [3]. The aims of PROA included improving the clinical results of patients with infections, to minimize the adverse events associated to the use of antibiotics including the emergence and spread of antibiotic resistance, and to ensure the use of the most costefficacious treatments, but also to provide guidelines for the implementation of these programs in Spanish hospitals. In the framework of these recommendations, a program for optimizing the use of antibiotics was implemented in a regional hospital. This study was designed to compare antibiotic consumption and costs, cost per process, mean hospital stay, and percentage of hospital readmissions before and after implementation of the program.

\section{Methods}

A quasi-experimental prospective intervention study for optimizing antibiotic use in the in-patient setting with a historical control group was designed. The study was conducted in hospital San Eloy in Barakaldo, Spain. This is a 128-bed regional hospital, with a reference population of 135,172 inhabitants that belongs to the Integrated Health Care Organization Barakaldo-Sestao from the public health care system (Osakidetza) of the Basque Country. In September 2013, a multidisciplinary team including specialists in internal medicine, preventive medicine and public health, microbiology, and pharmacy designed a program for optimizing antibiotic use based on the principles of PROA consensus [3] and adapted to the characteristics of the hospital and to material and human resources available. The study was approved by the Commission of Infections and Antibiotic Policy (CIPA) and by the Direction-Management of the hospital.

The program was based on a non-restrictive intervention model to help prescription, the optimization audits were performed by internal medicine specialists in all patients who met at least one criterion for inclusion. Inclusion criteria were as follows: any patient in which antibiotics of restricted use by CIPA (echinocandins, daptomycin, 
Citation: Ugalde-Espiñeira J, Bilbao-Aguirregomezcorta J, Sanjuan-López AZ (2016) A Program for Optimizing the Use of Antibiotics in the Hospital Setting: Experience in a Spanish Regional Hospital. J Antimicrob Agents 2: 128. doi:10.4172/2472-1212.1000128

Page 2 of 3

linezolid, and tigecycline) have been prescribed; patients with antibiotic prescription longer than 6 days; antimicrobial interactions detected; patients treated with drugs susceptible of dose adjustment according to the renal function; and all patients in which with more than one antibiotic had been prescribed. Patients admitted to the special care unit were excluded.

\begin{tabular}{|c|c|c|c|c|c|c|c|c|c|c|}
\hline & \multicolumn{5}{|c|}{ Pharmaceutical expenditure } & \multicolumn{5}{|c|}{ DDD/100 stays } \\
\hline & $\begin{array}{l}\text { First } \\
\text { period }\end{array}$ & Second period & Percentage & $Z$ value & $P$ value & $\begin{array}{l}\text { First } \\
\text { period }\end{array}$ & $\begin{array}{l}\text { Second } \\
\text { period }\end{array}$ & Percentage & $Z$-value & $\begin{array}{l}\text { P- } \\
\text { value }\end{array}$ \\
\hline Tetracyclines & $1,118.30$ & $1,418.70$ & 26.86 & -0.447 & 0.655 & 0.15 & 0.21 & 40 & -1.414 & 0.157 \\
\hline Penicillins & $11,334.10$ & $8,322.20$ & -26.57 & -1.363 & 0.173 & 29.83 & 27.85 & -6.64 & -1.153 & 0.249 \\
\hline Cephalosporins & $2,822.90$ & $2,922.90$ & 3.54 & -0.28 & 0.779 & 11.86 & 12.52 & 5.56 & -1.123 & 0.261 \\
\hline Carbapenems/ & \multirow{2}{*}{$30,952.50$} & \multirow{2}{*}{$26,023.20$} & \multirow{2}{*}{-15.93} & \multirow{2}{*}{-0.73} & \multirow{2}{*}{0.465} & \multirow{2}{*}{8.4} & \multirow{2}{*}{7.58} & \multirow{2}{*}{-9.76} & \multirow{2}{*}{-1.095} & \multirow{2}{*}{0.273} \\
\hline monobactams & & & & & & & & & & \\
\hline Sulfonamides ${ }^{*}$ & 61.8 & 102.7 & 66.33 & & & 0.55 & 0.57 & 3.64 & & \\
\hline Macrolides & 259.9 & 525.3 & 102.09 & -0.535 & 0.593 & 1.12 & 1.09 & -2.68 & 0 & 1 \\
\hline Anaerobicides & 684 & 437.7 & -36.01 & -1.342 & 0.18 & 2.94 & 1.91 & -35.03 & -1.342 & 0.18 \\
\hline Aminoglycosides & $1,009.70$ & 712.3 & -29.45 & -1.604 & 0.109 & 2.69 & 1.85 & -31.23 & -1.604 & 0.109 \\
\hline Quinolones & $1,659.60$ & $1,581.80$ & -4.69 & -1.604 & 0.109 & 11.85 & 13.65 & 15.19 & -0.816 & 0.414 \\
\hline Glycopeptides & $5,279.70$ & $6,028.20$ & 14.18 & -0.447 & 0.655 & 1.94 & 1.79 & -7.73 & -0.447 & 0.655 \\
\hline Antifungals & $11,170.90$ & $16,285.50$ & 45.78 & -0.365 & 0.715 & 3.42 & 1.66 & -51.46 & -0.73 & 0.465 \\
\hline Antivirals $^{\dagger}$ & 107.3 & 67.3 & -37.27 & -1 & 0.317 & 0.07 & 0.06 & -14.29 & -1 & 0.317 \\
\hline Antimycobacterial & 48.7 & 32.3 & -33.73 & -0.314 & 0.753 & 0.36 & 0.42 & 15.56 & -0.105 & 0.917 \\
\hline Colistin/ & & & & & & & & & & \\
\hline Nitrofurantoin & 100.5 & (1.5 & -04.10 & -0.442 & .000 & 0.1 & 0.14 & 40 & $0.44 t$ & 0.000 \\
\hline Other antibacterials & $31,715.30$ & $25,388.10$ & -19.95 & -0.535 & 0.593 & 2.91 & 2.5 & -14.09 & -0.535 & 0.593 \\
\hline Total & $98,330.90$ & $89,886.60$ & -8.59 & -1.964 & 0.049 & 78.19 & 73.8 & -5.61 & -1.342 & 0.18 \\
\hline
\end{tabular}

Table 1: Indicators of antibiotic consumption before and after implementation of the PROA program.

Variables assessed included antibiotic consumption (expressed as defined daily dose [DDD] per 100 patient stays) and costs, cost per process, mean hospital stay, and percentage of hospital readmissions. Data obtained during the 6-month study period (November 2013April 2014) were compared with data collected between November 2012 and April 2013. Data were analyzed with the SPSS statistical program. Changes in the study variables between the two periods were compared with the Wilcoxon signed-rank test. Statistical significance was set at $\mathrm{P}<0.05$.

\section{Results}

During the study period, a total of 307 audits in 209 patients (56.5\% men, median age 78 years [range 21-98]) were performed. Intervention of the PROA team was recorded on the $7^{\text {th }}$ day of treatment in $67.8 \%$ of cases, before the $7^{\text {th }}$ day in $17.9 \%$, and after the $7^{\text {th }}$ day in $14.3 \%$. In $65.8 \%$ of cases, antibiotic treatment was discontinued between the $7^{\text {th }}$ and the $10^{\text {th }}$ day. The most common drugs involved in audits were amoxicillin/clavulanate (14\%), piperacillin/tazobactam (13.3\%), and imipenem/cilastatin (11.4\%). Lack of indication (14.7\%) and completeness of treatment (43.6\%) were the most frequent causes of treatment withdrawal.

The reduction of pharmaceutical expenditure in antibiotics was $8.59 \%(\mathrm{P}=0.049)$ and the consumption in $\mathrm{DDD} / 100$ stays decreased by $5.61 \%$ (Table 1 ). The costs by processes in general surgery showed a $3.14 \%$ decrease $(\mathrm{P}=0.001)$. The mean length of stay was 3.66 days before implementation of the program and 3.94 days at 6 months after implementation of the program $(\mathrm{P}=0.594)$. The percentage of readmissions to the hospital decreased from $5.7 \%$ to $4.5 \%(\mathrm{P}=0.249)$.

\section{Discussion}

The present study shows a reduction in the consumption of antibiotics and pharmaceutical expenditures for these drugs as well as a favorable trend in the costs by processes after implementation of a 
Citation: Ugalde-Espiñeira J, Bilbao-Aguirregomezcorta J, Sanjuan-López AZ (2016) A Program for Optimizing the Use of Antibiotics in the Hospital Setting: Experience in a Spanish Regional Hospital. J Antimicrob Agents 2: 128. doi:10.4172/2472-1212.1000128

Page 3 of 3

program for optimizing the use of antibiotics in a medium-sized regional hospital in the Basque Country, Spain. In the PROA program, audits were performed on the $7^{\text {th }}$ day of treatment because this time allowed assessing discontinuation of antibiotic therapy in most infectious syndromes. According to data reported in our studies [4-7], audits could be started in our hospital on the $3^{\text {rd }}$ day of therapy in order to achieve an early optimization of the antimicrobial (indication, decrease of spectrum, sequential therapy) and reduction in the duration of treatment.

After implementation of PROA, there was a decrease in the consumption of antifungals, anaerobicides, and aminoglycosides. A reduction of $5.61 \%$ in $\mathrm{DDD} / 100$ stays was observed, although this percentage is lower than $13.82 \%$ reported by López-Medrano et al. [8] following application of a non-compulsory control program in the hospitalization units of six medical and surgical departments from a tertiary care hospital in Madrid, Spain. These differences may be explained by the characteristics of the hospital and the duration of the program (6 months vs. 12 months). Also, there was an $8.59 \%$ reduction $(8,444.25 €)$ in antibiotic expenditure. Our data regarding expenditures and consumption are lower than those found in other studies $[9,10]$, probably in relation to hospital-related characteristics, short time of the study, and use of less restrictive interventions. Other factors including commercialization of generic drugs or arrangements with the pharmaceutical companies may influence upon acquisition costs.

About $40 \%$ of audits of the program were performed in the service of general surgery, where internists of the PROA program also provided support in medical complications developed by surgical patients. It was found a significant decrease of the cost by process at the level of this service. This may be useful as a reference point for further comparisons and for the development of improvement strategies.

Limitations of the study include the impossibility to provide clinical indicators or outcome results, such as mortality related to sepsis or to infection by methicillin-sensitive Staphylococcus aureus, or to assess changes in resistant microorganisms. Despite these limitations, this study shows the feasibility of a program for optimizing the use of antibiotics in a regional hospital and the benefits associated with this strategy, which have been already remarkable after a short period of 6 months.
Conflicts of interest: None to be disclosed.

Funding: None

\section{References}

1. Lee CR, Cho IH, Jeong BC, Lee SH (2013) Strategies to minimize antibiotic resistance. Int J Environ Res Public Health 10: 4274-4305.

2. Cantón R, Horcajada JP, Oliver A, Garbajosa PR, Vila J (2013) Inappropriate use of antibiotics in hospitals: the complex relationship between antibiotic use and antimicrobial resistance. Enferm Infecc Microbiol Clin 4: 3-11.

3. Rodríguez-Baño J, Paño-Pardo JR, Alvarez-Rocha L, Asensio A, Calbo E, et al. (2012) Programs for optimizing the use of antibiotics (PROA) in Spanish hospitals: GEIH-SEIMC, SEFH and SEMPSPH consensus document. Enferm Infecc Microbiol Clin 30: 22.

4. Storey DF, Pate PG, Nguyen AT, Chang F (2012) Implementation of an antimicrobial stewardship program on the medical-surgical service of a 100-bed community hospital. Antimicrob Resist Infect Control 1: 32.

5. Fukuda T, Watanabe H, Ido S, Shiragami M (2014) Contribution of antimicrobial stewardship programs to reduction of antimicrobial therapy costs in community hospital with 429 beds -before-after comparative two-year trial in Japan. J Pharm Policy Pract 7: 10.

6. Braykov NP, Morgan DJ, Schweizer ML, Uslan DZ, Kelesidis T, et al. (2014) Assessment of empirical antibiotic therapy optimisation in six hospitals: an observational cohort study. Lancet Infect Dis 14: 1220-1227.

7. Moehring RW, Sloane R, Chen LF, Smathers EC, Schmader KE, et al. (2013) Delays in appropriate antibiotic therapy for gram-negative bloodstream infections: a multicenter, community hospital study. PLoS One 8.

8. López-Medrano F, San Juan R, Serrano O, Chaves F, Lumbreras C, et al. (2005) Impact of a non-compulsory antibiotic control program (PACTA): cost reductions and decreases in some nosocomial infections. Enferm Infecc Microbiol Clin 23: 186-190.

9. Pate PG, Storey DF, Baum DL (2012) Implementation of an antimicrobial stewardship program at a 60-bed long-term acute care hospital. Infect Control Hosp Epidemiol 33: 405-408.

10. Standiford HC, Chan S, Tripoli M, Weekes E, Forrest GN (2012) Antimicrobial stewardship at a large tertiary care academic medical center: cost analysis before, during, and after a 7-year program. Infect Control Hosp Epidemiol 33: 338-345. 